\title{
Acupuncture-Induced Pain Relief and Salivary Hormone Changes in Men and Women
}

\author{
Ilaria Ceccarelli1, Fu Bao Tian², Stefano Pieretti³, Paola Minosi³, Samuele Paparo Barbaro², \\ Paolo Fiorenzani' ${ }^{1}$ Jessica Pinassi ${ }^{1}$, Anna Maria Aloisi ${ }^{*}$ \\ ${ }^{1}$ Dipartimento Scienze Mediche, Chirurgiche e Neuroscienze, Università di Siena, Siena, Italy \\ ${ }^{2}$ Dipartimento di Medicina Clinica e Molecolare, Università La Sapienza, Rome, Italy \\ ${ }^{3}$ Centro Nazionale Ricerca e Valutazione Preclinica e Clinica dei Farmaci, Istituto Superiore di Sanità, Rome, Italy \\ Email: ^annamaria.aloisi@unisi.it
}

How to cite this paper: Ceccarelli, I., Tian, F.B., Pieretti, S., Minosi, P., Barbaro, S.P., Fiorenzani, P., Pinassi, J. and Aloisi, A.M. (2020) Acupuncture-Induced Pain Relief and Salivary Hormone Changes in Men and Women. Open Journal of Endocrine and Metabolic Diseases, 10, 29-43.

https://doi.org/10.4236/ojemd.2020.103005

Received: November 29, 2019

Accepted: March 1, 2020

Published: March 4, 2020

Copyright $\odot 2020$ by author(s) and Scientific Research Publishing Inc. This work is licensed under the Creative Commons Attribution International License (CC BY 4.0).

http://creativecommons.org/licenses/by/4.0/

\begin{abstract}
Objective: The aim of the present study was to examine the efficacy of acupuncture in the treatment of pain together with the determination of different hormonal parameters for possible correlations in men and women of different ages. Methods: 39 women and 38 men suffering from pain and seeking acupuncture received acupuncture treatment twice a week for 5 weeks. Pain and quality of life parameters were collected via written questionnaires (VAS, QUID and SF-36), while testosterone and cortisol were determined in the saliva. Results: Cortisol and testosterone salivary levels were higher in controls than in pain subjects and higher in men than in women. Acupuncture treatment improved all parameters, decreased pain and changed the hormonal values differently depending on sex and age. Conclusions: Sex and age are important factors in changing the effects of acupuncture in the treatment of pain.
\end{abstract}

\section{Keywords}

Pain, Acupuncture, Hormones, Saliva

\section{Introduction}

Acupuncture is an ancient Chinese treatment during which needles are inserted into acupuncture points. Acupuncture is believed to have the function of regulating the energy (Qi) flow and removing blood stasis by needling at specific acupuncture points. Various hypotheses have been advanced to explain its actions. Functional magnetic resonance imaging (fMRI) showed that acupuncture affects the activity of the limbic system and limbic-associated structures [1] [2] 
able to play a primary role in regulating emotion, attention, learning and memory. These brain areas present several anatomical and physiological sex differences [3]. Indeed, research has shown sex differences in the response to acupuncture treatment [1] [4].

Acupuncture has become a widespread therapy for many pathological conditions, such as infertility [5] [6], headache [7], obesity [8], depression [9], and painful conditions of different origin and localization [10]. Chronic pain affects a large percentage of the population in all civilized countries, with high personal, familial and economic burdens. Most chronic pain syndromes are more frequent in women, who often report higher levels of pain intensity and frequency than men [11]. Several reasons for these differences have been proposed. One of the most intriguing relates to gonadal hormones, which vary considerably in the two sexes and at different ages. Endocrine glands produce hormones under the direction of various factors, from the brain, in which the nervous or endocrine stimulus is elaborated, to the target gland; indeed, their activity can be affected by light, temperature, food, etc. [12]. Acupuncture was found to increase estrogen receptor (ER)- $\beta$ decrease ER- $\alpha$ and modulate $17 \beta$-estradiol in rats [13].

The involvement of steroid hormones in pain modulation has been demonstrated [14], with studies suggesting the involvement of estrogens and testosterone in pain modulation via several pathways. We have repeatedly shown that estradiol and testosterone can increase/decrease pain in rats and humans depending on the sex and "experimental" conditions [15] [16]. In the present study, we have focused on cortisol and testosterone because they are involved in all body functions, often differently in male and female subjects [14] [17] [18] [19] [20]. Cortisol is certainly involved in inflammation and pain because it is well known that high levels of cortisol have an important anti-inflammatory and hyperglycemic action to help the subject recover [19]. Cortisol is a steroid hormone derived from cholesterol, it is present in the blood usually bound to corticosteroid-binding globulin, with only a very small amount unbound. The unbound cortisol (free fraction) is the biologically active fraction able to pass through the acinar cells to enter the saliva via passive diffusion [21]. As for Testosterone, in both sexes, it increases red cell production and muscle tone, has an antidepressant function and was shown to play an analgesic role [20] [22]. Testosterone, also derived from cholesterol, is mainly produced by the testes in men, with only a minor contribution from the adrenals. In women, testosterone is produced by the ovaries and the adrenal glands, a source particularly important after menopause. Testosterone is also considered a pro-hormone since its effects are mediated by two main products: DHT (dihydrotestosterone, after reduction by $5 \alpha$-reductase) and estradiol (after aromatization by aromatase). Testosterone is supplied to target tissues by the blood where much of it is transported bound to a specific plasma protein, sex hormone-binding globulin (SHBG). It is known that an age-related increase in SHBG attenuates the age trend in total serum testosterone but not that in serum free testosterone or salivary testosterone [23]. Salivary testosterone should represent the bioavailable 
hormone levels, which are unaffected by changes in the SHBG [24]. Overall, these hormones are key factors in the determination of body health.

There are many advantages of the use of saliva for hormone biomarker assessment [25]. Salivary determinations are a good alternative to the collection of serum, which is always invasive and more expensive. Saliva collection is relatively easy and can be replicated when needed. Moreover, salivary hormone determination is known to reflect the free or biologically active hormone fraction [21].

The aim of the present study was to evaluate the effect of a cycle of acupuncture treatment on salivary cortisol and testosterone levels in men and women suffering from pain. Questionnaires were administered to evaluate the pain condition and health status.

\section{Materials and Methods}

\subsection{Subjects}

39 female and 38 male pain subjects were recruited among the outpatients of Dr. Bao Tian Fu (Arezzo, Italy). Subjects were included if they provided written informed consent to take part in the study, which was conducted in accordance with the Declaration of Helsinki. In particular, inclusion criteria: presence of pain, agreement to fill questionnaires, agreement to give saliva sample; exclusion criteria: do not sign the informed consent. A single licensed acupuncturist (BTF), in clinical practice for over 25 years, administered the acupuncture to all the pain patients, who were treated according to their primary pathology. In particular, after the patients were relaxed, the acupuncturist sterilized the skin around the acupuncture point and then inserted stainless steel needles (SUNRISE, Qui Tian, San Marino) into the acupuncture points considered significant for that pathology, the lunar phase and the time of day. Treatment consisted of 10 sessions carried out twice a week. All measures were collected three times: at baseline before the $1^{\text {st }}$ session (Test I), before the $5^{\text {th }}$ session (Test II) and before the $10^{\text {th }}$ session (Test III). The subjects provided the saliva sample in the morning between 9:00-11:00 AM. No food was taken 90 min beforehand.

To compare the basal conditions of the pain patients with those of non-pain subjects, 12 age-matched females and 13 males were recruited from the general population.

\subsection{Saliva Collection}

Saliva samples were collected using the Salivette collection device (Sarstedt Inc., Nuembrecht, Germany). The subjects took a cotton wool tamponade out of a small tube, placed it in their mouth, chewed on it for $30-45 \mathrm{sec}$, and then put it back in the tube. Samples were centrifuged and stored at $-20^{\circ} \mathrm{C}$ until hormone determination, carried out using commercially available kits.

\subsection{Hormone Assay}

Samples were assayed in duplicate for salivary cortisol and testosterone with 
ELISA kits based on competitive binding, without modification of the manufacturer's recommended protocols (Demeditec, Kiel, Germany). For cortisol, the sensitivity was $0.024 \mathrm{ng} / \mathrm{ml}$, the intra-assay variation was $4.8 \%$ and the inter-assay variation was $6.3 \%$. For testosterone, the sensitivity was $2.2 \mathrm{pg} / \mathrm{ml}$, the intra-assay variation was $7.2 \%$ and the inter-assay variation was $9.2 \%$.

\subsection{Questionnaires}

Visual Analog Scale (VAS). Subjects were asked to indicate the pain level experienced in the last few days on a scale from 0 (no pain) to 10 (maximum pain).

Present Pain Intensity (PPI). Subjects were asked to indicate their actual pain level on a scale from 0 (no pain) to 5 (maximum pain).

\subsection{QUID}

The Italian Pain Questionnaire (QUID), a reconstructed Italian version of the McGill Pain Questionnaire [26], consists of 42 descriptors divided into four pain rating index ranks (sensory, affective, evaluative and mixed). The Total Pain Rating Index rank value (PRI-t), given by the sum of all the rank values, describes and quantifies pain.

\subsection{Short Form-36 (SF-36)}

The Italian version of the SF-36 questionnaire [27] is a generic multidimensional instrument for assessing quality of life. It consists of 36 items grouped into two components and divided into eight scales: The Physical Component Summary (PCS-36) encompasses physical functioning (PF), role physical (RP), body pain (BP), general health (GH); the Mental Component Summary (MCS-36), which refers to vitality (VT), includes social functioning (SF), role emotional (RE) and mental health $(\mathrm{MH})$. Individual items are scored on a 0 - 100 standardized Likert scale. For each domain, including BP and the summary components PCS and MCS, a higher score indicates a better quality of life and lower limitations.

\subsection{Statistical Analysis}

To evaluate the differences between pain subjects (PAIN) and controls (CTR), the basal levels of salivary hormones (cortisol and testosterone) and the status of perceived health (SF-36) were evaluated by three-way ANOVA with Sex (2 levels: men and women), Age (2 levels, $<50$ and $>50$ ) and Group (2 levels, CTR and PAIN) as factors. The age groups were defined according to women in reproductive years $(<50)$ and in menopause $(>50)$.

To test the acupuncture-induced effects, the data from the PAIN group were analyzed in the three determinations carried out (Test I, Test II, Test III). Three-way ANOVA with the factors Sex (2 levels: men and women), Age (2 levels, $<50$ and $>50$ ) and Test ( 3 levels, Test I-III, repeated) was performed.

\section{Results}

A summary of the subjects' data is presented in Table 1 . In both sexes, pain con- 
sisted mostly of diffuse musculoskeletal pain.

\subsection{Comparison between CTR and PAIN Groups (Test I)}

Data were analyzed with a three-way ANOVA with the factors Sex, Age and Group.

\section{HORMONES: Cortisol and Testosterone}

ANOVA applied to cortisol levels (Figure 1(A), Figure 1(A')) revealed a significant effect of Sex $(F(1,94)=6.13, p<0.015)$ and $\operatorname{Group}(F(1,94)=12.25, p<$

Table 1. Summary of groups, sex, number of patients per group and kind of pain.

\begin{tabular}{cccc}
\hline Groups & Sex & Number of patients & Pain \\
\hline Control & Male & 13 & No pain \\
Control & Female & 12 & No pain \\
& Male & 38 & $\mathrm{~N}=6$ neuropathic pain \\
Pain & & $\mathrm{N}=24$ diffuse muscle pain \\
& & $\mathrm{N}=8$ headache \\
& Female & 39 & $\mathrm{~N}=11$ neuropathic pain \\
Pain & & $\mathrm{N}=19$ diffuse muscle pain \\
& & N = 9 headache \\
\hline
\end{tabular}

\section{CORTISOL BASAL LEVELS}

A

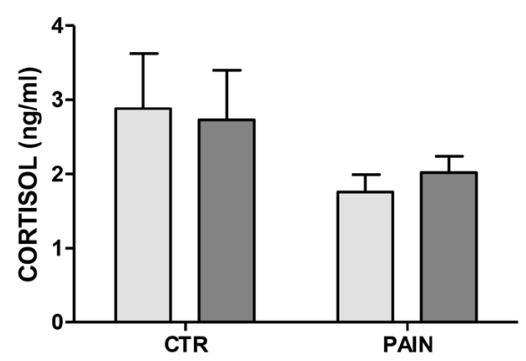

$A^{\prime}$

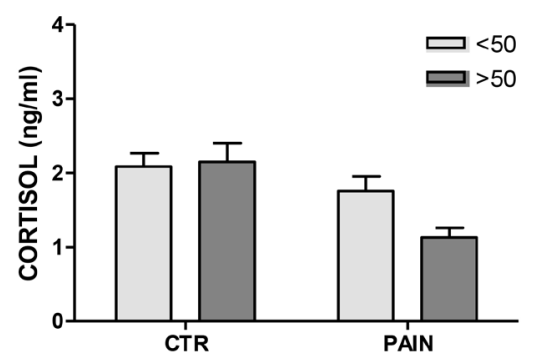

TESTOSTERONE BASAL LEVELS
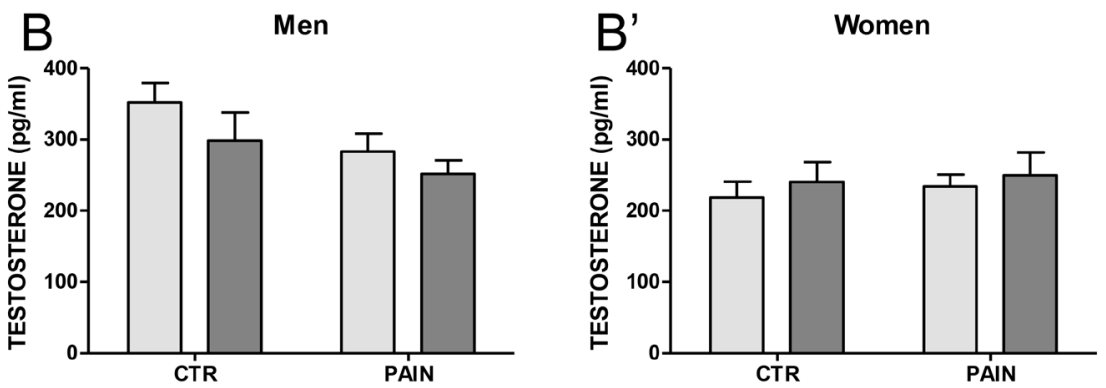

Figure 1. Cortisol and testosterone basal levels in pain and control subjects. Cortisol (A, $\mathrm{A}^{\prime}$ ) and testosterone (B, B') basal levels determined in the saliva of pain and control subjects (male and female, younger and older than 50 years). Data are means \pm SEM. 
0.001). These results were due to the generally higher cortisol levels in men than in women and the higher levels in the CTR group than in the PAIN group, independently of Age. ANOVA applied to testosterone levels (Figure 1(B), Figure $\left.1\left(B^{\prime}\right)\right)$ showed a significant effect of $\operatorname{Sex}(F(1,94)=7.1903, p<0.001)$, with lower levels in the female subjects than in the male ones, independently of Group or Age.

\section{QUESTIONNAIRES: SF-36}

All the subscales of the SF-36 (PF, RP, GH, VT, SF, RE, MH, BP as well as the Mental and Physical Component Summary: MCS-36 and PCS-36) showed a significant effect of Group ( $\mathrm{p}<0.001$ for all) (Figure 2). PAIN groups had lower scores than CTR ones, regardless of Age and Sex, indicating a worse perception of their health status ( $\mathrm{p}<0.001$ for all).

\subsection{Analysis of Test I, Test II and Test III in Pain Patients}

To evaluate changes during acupuncture treatment, three-way ANOVA with the factors Sex, Age and Test was carried out only in subjects that participated in all three tests, i.e. 23 males $(\mathrm{n}=11<50, \mathrm{n}=12>50)$ and 21 females $(\mathrm{n}=11<50, \mathrm{n}$ $=10>50)$. In particular, Figure 2 shows cortisol and testosterone levels determined

\section{SF-36 basal levels}

\section{MEN}

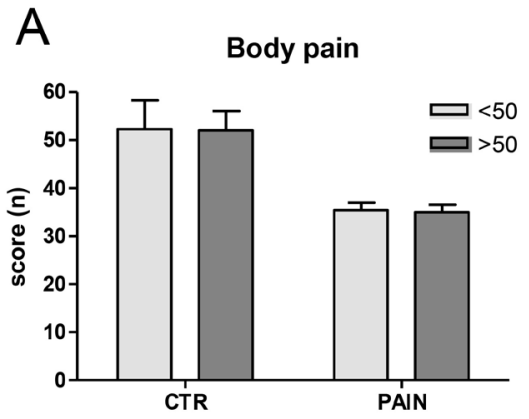

B

MCS

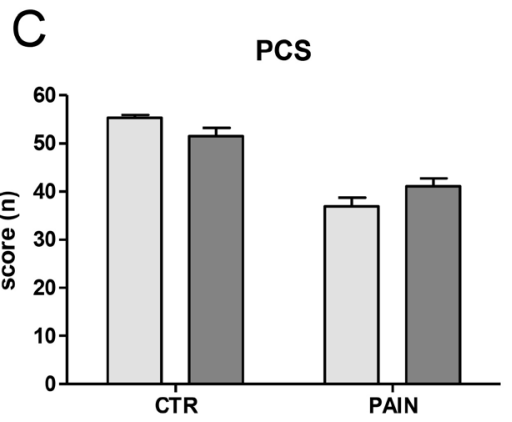

WOMEN
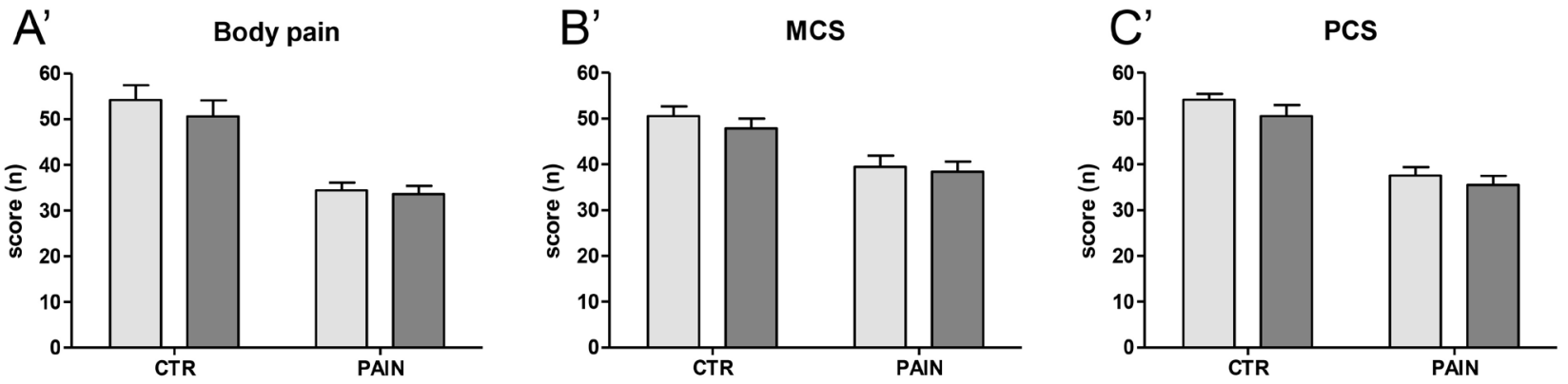

Figure 2. SF-36 basal data recorded in pain and control subjects. SF-36 basal data recorded in pain and control subjects (male and female, younger and older than 50 years). Data are means \pm SEM. Body Pain (A, A'); MCS: Mental Component Summary (B, B'); PCS: Physical Component Summary (C, C'). 
in the saliva and SF-36 data of men and women pain patients at the 1st, 5th and 10th acupuncture treatments.

\section{HORMONES: Cortisol and Testosterone}

Cortisol (Figure 3(A), Figure 3(A')). ANOVA showed a significant effect of Age $(\mathrm{F}(1,36)=5.77, \mathrm{p}<0.021)$ due to the lower levels in older subjects than in younger ones of both sexes. In particular, older women had the lowest levels at the first determination, which increased at Test II and remained at this higher level at Test III. In contrast, the younger women had higher values $(p=0.05)$ at Test I, which decreased at Test II and returned to the basal level at Test III. In the men, the levels did not change in the younger group, but tended to decrease in the older group $(\mathrm{p}=0.05)$.

Testosterone (Figure 3(B), Figure 3(B')). ANOVA demonstrated a significant Sex $\times$ Age $\times$ Test interaction $(F(2,70)=8.06, p<0.001)$. Younger males and

\section{CORTISOL LEVELS, TIME COURSE}
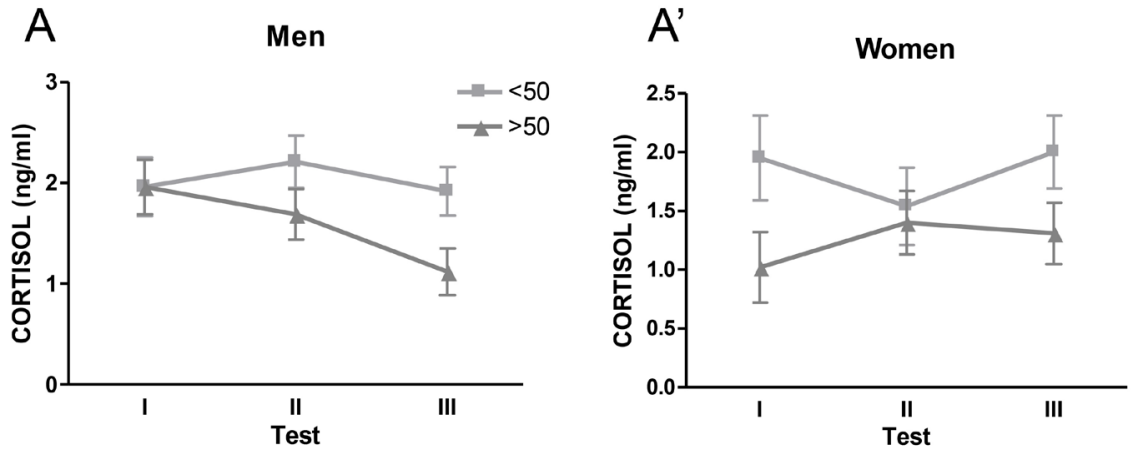

\section{TESTOSTERONE LEVELS, TIME COURSE}
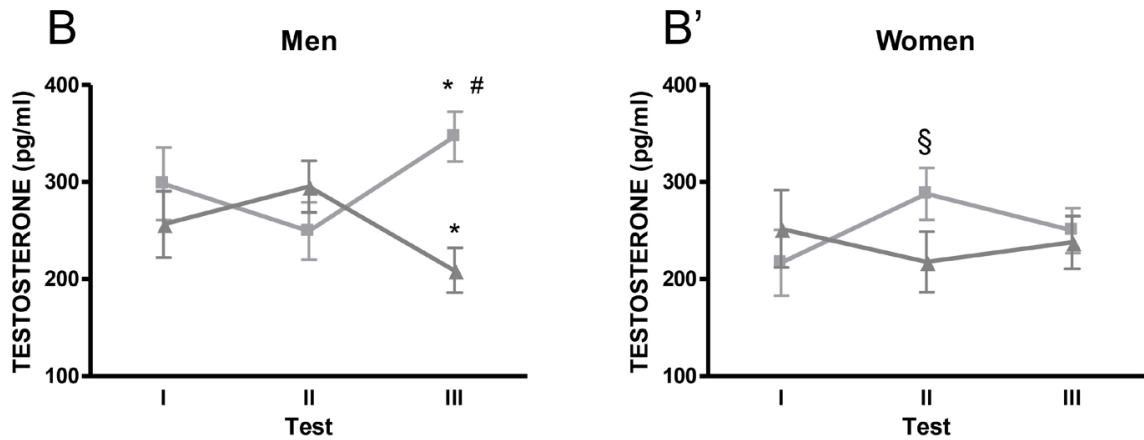

Figure 3. Time course of cortisol and testosterone levels during acupuncture treatment. Cortisol (A, A') and testosterone (B, B') levels determined in the saliva of male and female pain subjects at the 1st, 5 th and 10th acupuncture treatments (Test I-III). Data are given for subjects younger and older than 50 years and are expressed as means \pm SEM. ${ }^{*} \mathrm{p}<$ 0.01 , vs same group Test II, \#p $<0.001$ vs same sex Test III; $\$ p<0.01$, vs same group Test I. 
females displayed a significant increase during the treatment, with males having higher levels at Test III than at Test II $(\mathrm{p}<0.005)$ and females at Test II than at Test I $(\mathrm{p}<0.01)$. Older men had lower levels at Test III than at Test II $(\mathrm{p}<$ 0.004). Moreover, the testosterone level was significantly higher in younger males than in older ones at Test III $(\mathrm{p}<0.001)$. In the older women, no changes occurred during the repetitions of the acupuncture treatments.

\section{PAIN PARAMETERS}

Pain scores recorded in men and women pain patients at the 1st, 5th and 10th acupuncture treatments are presented in Figure 4.

\section{Visual Analog Scale (VAS)}

ANOVA applied to VAS scores (Figure 4(A), Figure 4(A')) revealed a significant effect of Test $(\mathrm{F}(2,64)=16.99, \mathrm{p}<0.001)$. In both men and women, the VAS scores decreased from Test I to Test III and the younger women tended to have lower scores than the older ones.

Total Pain Rating Index (PRI-t)

ANOVA applied to the PRI-t (Figure 4(B), Figure 4(B')), derived from the QUID analysis, revealed a significant effect of Test $(F(2,72)=7.39, p<0.001)$

\section{PAIN SCORE, TIME COURSE}
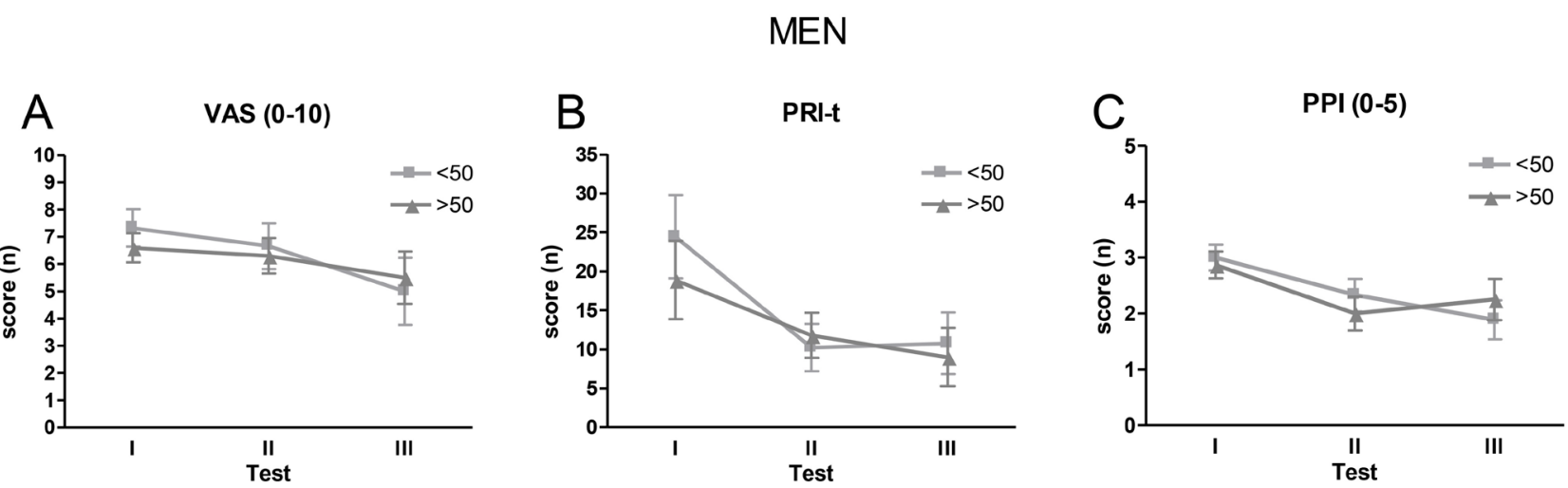

\section{WOMEN}
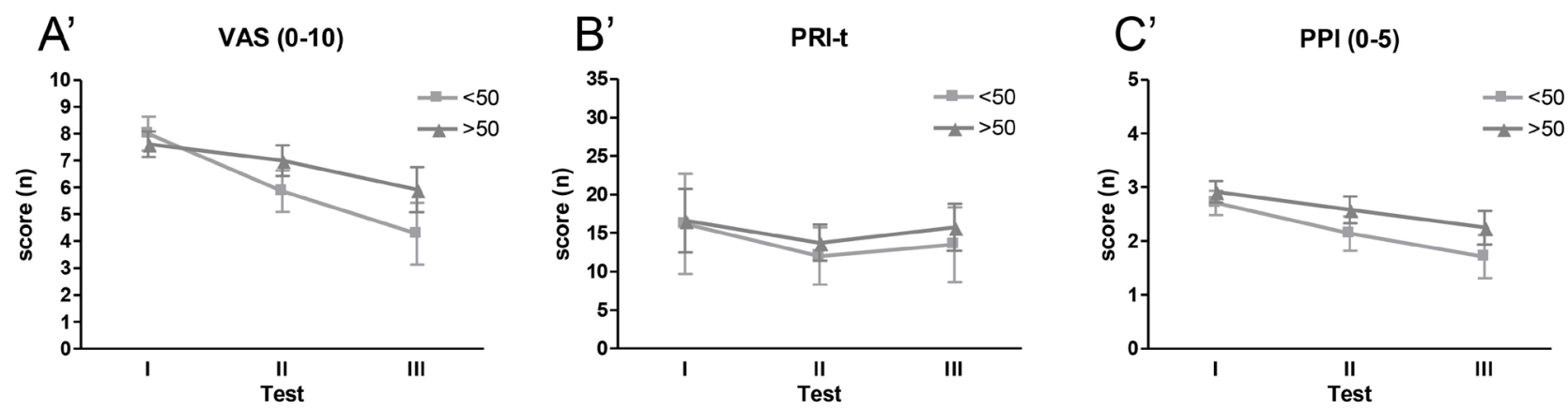

Figure 4. Time course of pain scores during acupuncture treatment. Pain scores recorded in male and female pain subjects at Test I-III. Data are expressed as means \pm SEM. VAS: Visual Analog Scale (A, A'); PRI-t: Total Pain Rating Index rank value (B, B'); PPI: Present Pain Intensity (C, C’). 
due to the progressive decrease of the score from Test I to Test III. Moreover, there was a significant Sex $\times$ Test interaction $(F(2,72)=3.06, \mathrm{p}<0.05)$ due to the higher levels in men than in women at Test I.

\section{Present Pain Intensity (PPI)}

ANOVA applied to PPI (Figure $4(C)$, Figure $4\left(C^{\prime}\right)$ ) revealed a significant effect of Test $(F(2,64)=11.54, p<0.001)$. The score decreased from Test I to Test III independently of Sex and Age.

\section{QUESTIONNAIRES: SF-36}

ANOVA applied to body pain (BP) (Figure 5(A), Figure 5( $\left.\mathrm{A}^{\prime}\right)$ ) showed a significant effect of Test $(\mathrm{F}(2,66)=8.93, \mathrm{p}<0.0004)$ due to the significant increase (i.e. improvement) from Test I to Test III, independently of Sex and Age. In the Mental Component Summary (MCS-36) parameter (Figure 5(B), Figure $\left.5\left(B^{\prime}\right)\right)$, ANOVA showed a significant Sex $\times$ Age interaction $(F(1,22)=4.20, p<$ $0.05)$. While older men tended to have higher scores than the younger ones, indicating that their condition was better, older women tended to have lower scores than younger ones. ANOVA applied to the Physical Component (PCS) of the SF-36 (Figure 5(C), Figure 5(C')) revealed a significant effect of Test (F (2, $44)=6.17, \mathrm{p}<0.004)$, as well as a significant Test $\times$ Age interaction $(\mathrm{F}(2,44)=$

\section{SF-36 TIME COURSE}

\section{MEN}
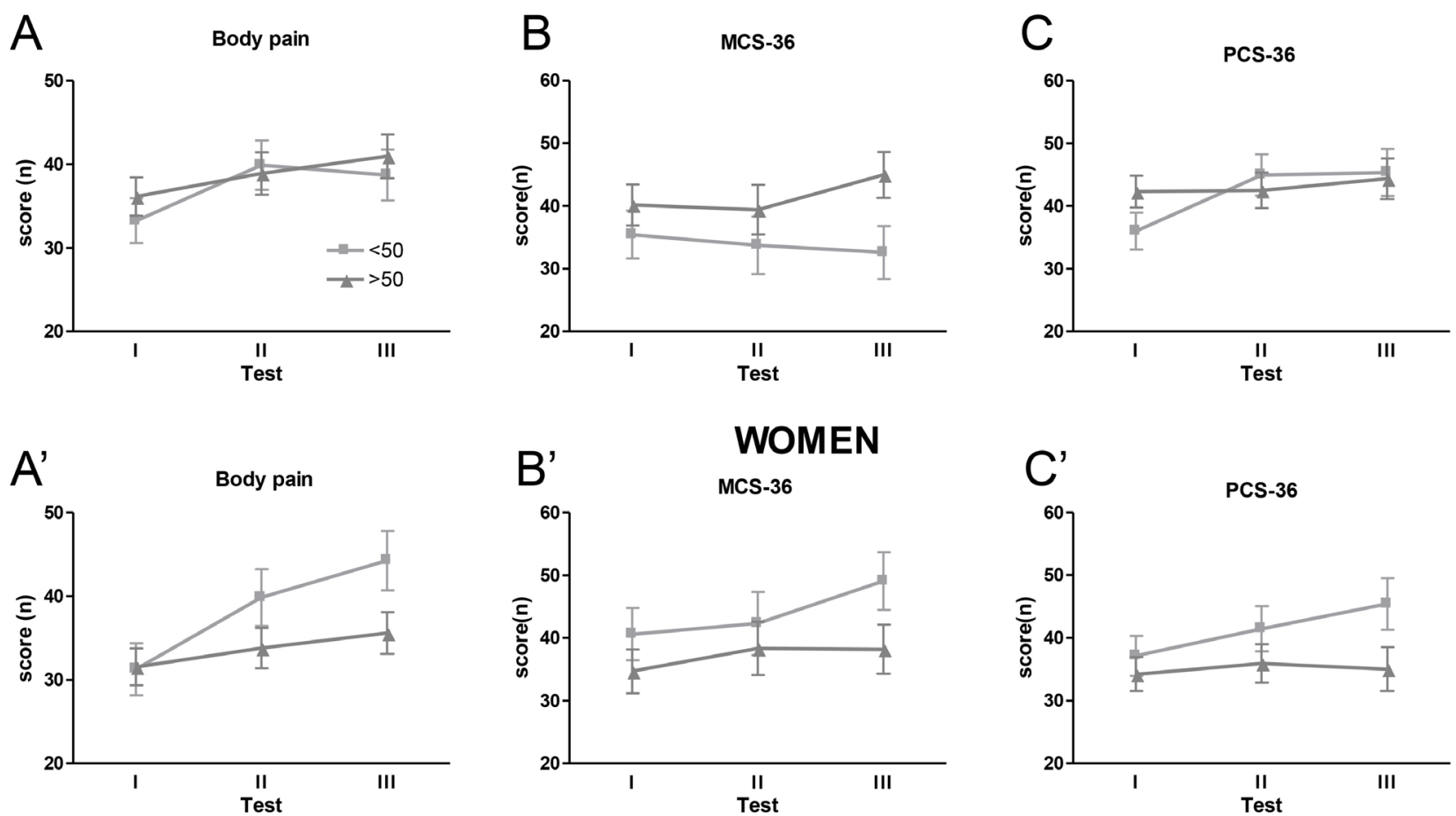

Figure 5. Time course of SF36 data during acupuncture treatment. SF36 data recorded in male and female pain subjects at Test I-III. Data are given for subjects younger and older than 50 years and are expressed as means \pm SEM. Body Pain (A, A'); MCS: Mental Component Summary (B, B’); PCS: Physical Component Summary (C, C’). 
$3.26, \mathrm{p}<0.05)$. Scores of younger subjects progressively increased from Test I to Test II to Test III ( $\mathrm{p}<0.005$ and $\mathrm{p}<0.0004$, respectively), whereas in older subjects there was no effect of treatment.

\section{QUID}

ANOVA applied to the sensorial (QUIDs), affective (QUIDa) and emotional (QUIDe) subscales of the QUID pain questionnaire (Figures 6(A)-(D), Figures $\left.6\left(A^{\prime}\right)-\left(D^{\prime}\right)\right)$ showed a significant effect of Test $(F(2,72)=5.05, p<0.009, F(2$, $72)=8.17 \mathrm{p}<0.001, \mathrm{~F}(2,72)=5.24, \mathrm{p}<0.007$, respectively) due to a progressive decrease from Test I to Test III. These effects were more evident in men in all the subscales. Moreover, in the sensorial component (QUIDs), a significant Sex $\times$ Test interaction $(\mathrm{F}(2,72)=3.55, \mathrm{p}<0.034)$ was due to lower levels in Test II and Test III than in Test I ( $\mathrm{p}<0.004$ and $\mathrm{p}<0.001$, respectively) in males but not in females.

\section{Discussion}

Acupuncture is an ancient Chinese procedure to treat many clinical conditions. Its ability to interact with body functions with practically no side effects has favored its spread to all parts of the world. Pain relief is one of the best known of the several beneficial effects of acupuncture treatment. In the present study, acupuncture-induced effects on pain were evaluated in men and women of different ages. The results clearly show sex and age differences in pain and hormonal changes.

\section{QUID SCORE, TIME COURSE}

\section{MEN}
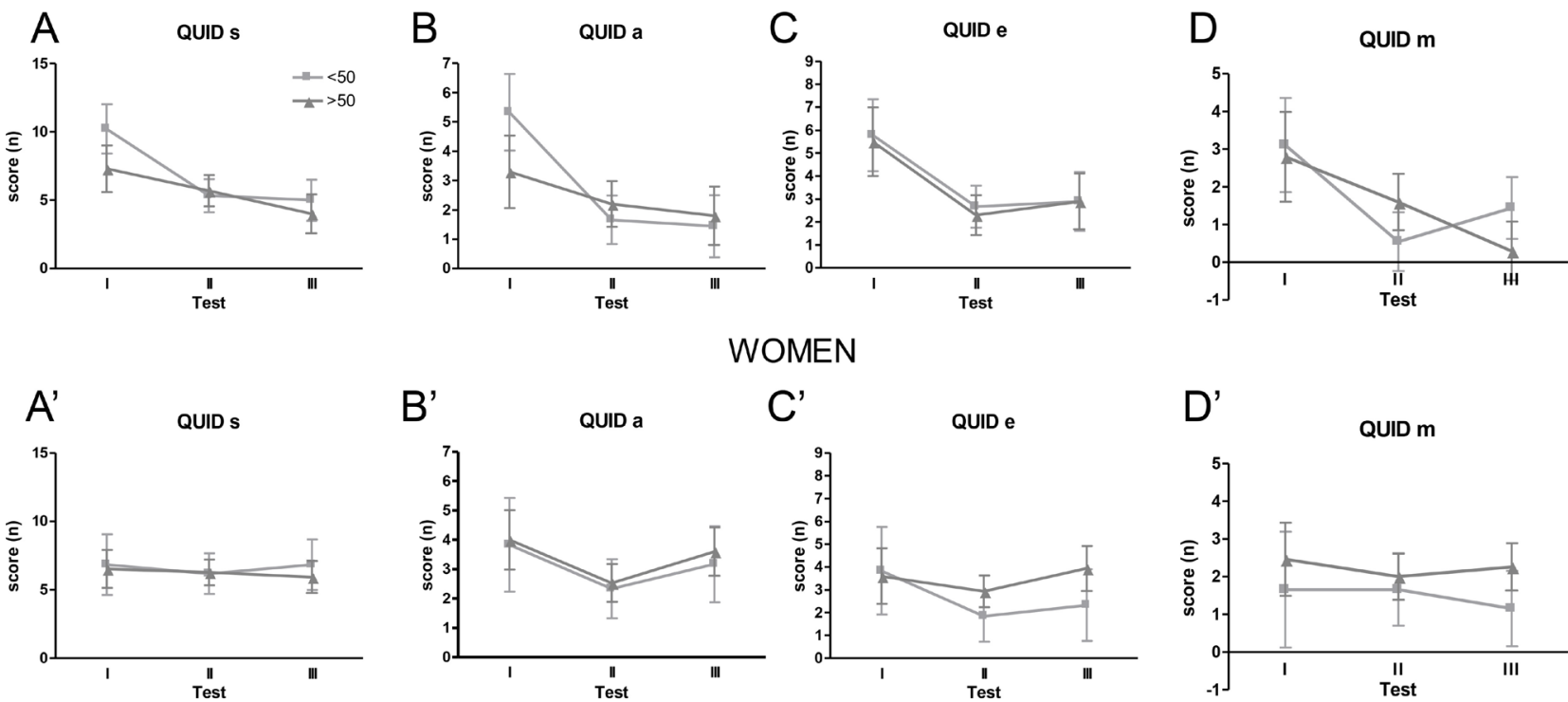

WOMEN

$\mathrm{C}^{\prime}$
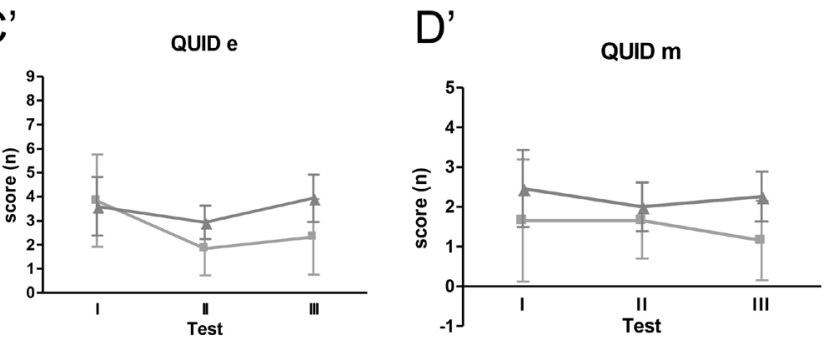

Figure 6. Time course of QUID scores during acupuncture treatment. Time course of the different QUID components: s: sensorial (A, A'), a: affective (B, B'), e: emotional (C, C'), m: miscellaneous (D, D'). All scores are recorded in male and female pain patients at Test I-III. Data are given for subjects younger and older than 50 years and are expressed as means \pm SEM. 
In the first part of the study, data from pain patients and controls were compared. Patients had lower levels than controls for cortisol (in both sexes) and testosterone (in men). Similarly, the scores of quality of life parameters (determined with the SF-36 questionnaire) were lower in both sexes and both age groups of pain patients. These results are in agreement with previous data showing that pain and/or analgesics can induce a strong decrease in steroid hormones such as cortisol and testosterone [28]. Thus, it appears that these hormones (i.e. their free fractions) are affected by the painful condition. Since it is very difficult in this kind of study to have patients that are similar in terms of painful condition and/or length of pain, drugs and/or previous treatments, the fact that all groups show the same results is indicative of a trend in which pain loses its ability to be stressful (as in acute pain) and the gonads and adrenals decrease their production, maintaining a hormonal impairment which, if prolonged, can affect physical and mental aspects. This is evident from the questionnaire results, with pain patients showing worse values than controls of the same age and sex.

Acupuncture treatment was able to improve pain and the other parameters in both sexes and at all ages. Although the pain intensity was initially very high in all pain groups, with Visual Analog Scale (VAS) scores of 6 - 7 in men and 7 - 8 in women, it decreased to scores of 5 - 6 in men and $4-7$ in women. Interestingly, pain changes were accompanied by changes in all parameters, albeit differently in the two sexes and in the two age groups.

In men, salivary cortisol values showed similar levels in both age groups at the beginning of treatment. Then, while in the young subjects, cortisol remained stable, in the older ones it tended to decrease. In women, while in the younger subjects the levels were comparable to men and tended to decrease or remain stable with treatment, in older women the initial low levels suggested an impairment that could be related to the chronicity of the illness [29], i.e. pain relief was accompanied by higher physiological levels of cortisol.

Cortisol is a product of the hypothalamic-pituitary-adrenal axis. In addition to basal activity related to body functions, especially glucose metabolism, its levels can change in response to any kind of stressors [30]. It appears that chronic pain does not suppress the adrenals in men as it does in women.

Testosterone is present in both sexes at all ages, and blood levels are higher in men than in women. Also in the present experiment, in which values were determined in the saliva, men showed higher levels than women. While in men the values appeared to be lower in pain patients than controls, in women the difference was not significant in both age groups. During treatment, testosterone changed from the first to the third determination only in men: the young subjects presented the highest levels at the third determination, while the older ones showed a significant decrease.

It would be interesting to better define the influence of acupuncture treatment on the HPA and HPG axes. The hormones involved in these axes have many 
functions able to affect stress, pain and several other conditions. Moreover, needle manipulation during acupuncture has sexually dimorphic effects not only on the brain regions related to sensation but also on the networks related to cognition and emotion [31]. These findings are not unexpected since gender differences in endogenous opioids and the serotoninergic and cholinergic systems are well known, supporting the idea that male and female brains are different [32].

\section{Conclusion}

It has been widely demonstrated that acupuncture can relieve pain. However, few data are available about the gender-related efficacy of the treatment and the role of age in the changes occurring during an acupuncture session. Indeed, the mixing of different data (men plus women, younger with older people) may hide positive results merely because not all groups change in the same direction. The present study clearly shows the need to consider the gender and age of subjects separately.

\section{Acknowledgements}

The authors thank the external and university staff involved in this research.

\section{Authors Contribution Statements}

IC, BTF, SP, PM, SPB, PF, JP, AMA: Substantial contributions to the conception or design of the work, or the acquisition, analysis or interpretation of the data.

IC, BTF, AMA: Drafting of the work or critical revision of its intellectual content.

IC, BTF, SP, PM, SPB, PF, JP, AMA: Final approval of the version to be published, and agreement to be accountable for all aspects of the work in ensuring that questions related to the accuracy or integrity of any part of the work are appropriately investigated and resolved.

\section{Competing Interests}

The authors declare that they have no competing interests.

\section{Ethics Approval and Consent to Participate}

Subjects were included if they provided written informed consent to take part in this study, which was conducted in accordance with the Declaration of Helsinki.

\section{Funding}

This research was supported by the University of Siena, the Sapienza University of Rome and ISS funds.

\section{Conflicts of Interest}

The authors declare no conflict of interest. 


\section{References}

[1] Yeo, S., Rosen, B., Bosch, P., Noort, M.V. and Lim, S. (2016) Gender Differences in the Neural Response to Acupuncture: Clinical Implications. Acupuncture in Medicine, 5, 364-372. https://doi.org/10.1136/acupmed-2015-011025

[2] Li, Q.Q., Shi, G.X., Xu, Q., Wang, J., Liu, C.Z. and Wang, L.P. (2013) Acupuncture Effect and Central Autonomic Regulation. Evidence-Based Complementary and Alternative Medicine, 2013, Article ID: 267959. https://doi.org/10.1155/2013/267959

[3] Xu, C., Li, C., Wu, H., Wu, Y., Hu, S., Zhu, Y., Zhang, W., Wang, L., Zhu, S., Liu, J., Zhang, Q., Yang, J. and Zhang, X. (2015) Gender Differences in Cerebral Regional Homogeneity of Adult Healthy Volunteers: A Resting-State FMRI Study. BioMed Research International, 2015, Article ID: 183074. https://doi.org/10.1155/2015/183074

[4] Lund, I. and Lundeberg, T. (2008) Is It All about Sex? Acupuncture for the Treatment of Pain from a Biological and Gender Perspective. Acupuncture in Medicine, 26, 33-45. https://doi.org/10.1136/aim.26.1.33

[5] Zhong, Y., Zeng, F., Liu, W., Ma, J., Guan, Y. and Song, Y. (2019) Acupuncture in Improving Endometrial Receptivity: A Systematic Review and Meta-Analysis. BMC Complementary and Alternative Medicine, 19, Article No. 61. https://doi.org/10.1186/s12906-019-2472-1

[6] El-Toukhy, T., Sunkara, S.K., Khairy, M., Dyer, R., Khalaf, Y. and Coomarasamy, A. (2008) A Systematic Review and Meta-Analysis of Acupuncture in in Vitro Fertilisation. BJOG: An International Journal of Obstetrics and Gynaecology, 115, 1203-1213. https://doi.org/10.1111/j.1471-0528.2008.01838.x

[7] Linde, K., Allais, G., Brinkhaus, B., Fei, Y., Mehring, M., Shin, B.C., Vickers, A. and White, A.R. (2016) Acupuncture for the Prevention of Tension-Type Headache. Cochrane Database of Systematic Reviews, 4, CD007587. https://doi.org/10.1002/14651858.CD007587.pub2

[8] Wang, L.H., Huang, W., Wei, D., Ding, D.G., Liu, Y.R., Wang, J.J. and Zhou, Z.Y. (2019) Mechanisms of Acupuncture Therapy for Simple Obesity: An Evidence-Based Review of Clinical and Animal Studies on Simple Obesity. Evidence-Based Complementary and Alternative Medicine, 2019, Article ID: 5796381. https://doi.org/10.1155/2019/5796381

[9] Tu, C.H., MacDonald, I. and Chen, Y.H. (2019) The Effects of Acupuncture on Glutamatergic Neurotransmission in Depression, Anxiety, Schizophrenia, and Alzheimer's Disease: A Review of the Literature. Frontiers in Psychiatry, 10, 14. https://doi.org/10.3389/fpsyt.2019.00014

[10] Patil, S., Sen, S., Bral, M., Reddy, S., Bradley, K.K., Cornett, E.M., Fox, C.J. and Kaye, A.D. (2016) The Role of Acupuncture in Pain Management. Current Pain and Headache Reports, 22, 1-8. https://doi.org/10.1007/s11916-016-0552-1

[11] Aloisi, A.M. (2017) Why We Still Need to Speak about Sex Differences and Sex Hormones in Pain. Pain and Therapy, 6, 111-114. https://doi.org/10.1007/s40122-017-0084-3

[12] Morris, C.J., Aeschbach, D. and Scheer, F.A. (2012) Circadian System, Sleep and Endocrinology. Molecular and Cellular Endocrinology, 349, 91-104. https://doi.org/10.1016/j.mce.2011.09.003

[13] Liu, L.J., Du, H.L., Jin, Y.C. and Chen, J.W. (2007) Effects of Combined Acupuncture and Medicine Therapy on the Breast Tissue and Expressions of Estrogen Re- 
ceptor Subgroups in the Rat of Hyperplasia of Mammary Glands. Chinese Acupuncture \& Moxibustion, 27, 279-283.

[14] Maurer, A.J., Lissounov, A., Knezevic, I., Candido, K.D. and Knezevic, N.N. (2016) Pain and Sex Hormones: A Review of Current Understanding. Pain Management, 6, 285-296. https://doi.org/10.2217/pmt-2015-0002

[15] Aloisi, A.M., Bachiocco, V., Costantino, A., Stefani, R., Ceccarelli, I., Bertaccini, A. and Meriggiola, M.C. (2007) Cross-Sex Hormone Administration Changes Pain in Transsexual Women and Men. Pain, 132, S60-S67. https://doi.org/10.1016/j.pain.2007.02.006

[16] Ceccarelli, I., Scaramuzzino, A., Massafra, C. and Aloisi, A.M. (2003) The Behavioral and Neuronal Effects Induced by Repetitive Nociceptive Stimulation Are Affected by Gonadal Hormones in Male Rats. Pain, 104, 35-47. https://doi.org/10.1016/S0304-3959(02)00460-8

[17] Chen, X., Zhang, J. and Wang, X. (2016) Hormones in Pain Modulation and Their Clinical Implications for Pain Control: A Critical Review. Hormones, 15, 313-320. https://doi.org/10.14310/horm.2002.1696

[18] Kudielka, B.M. and Kirschbaum, C. (2005) Sex Differences in HPA Axis Responses to Stress: A Review. Biological Psychology, 69, 113-132.

https://doi.org/10.1016/j.biopsycho.2004.11.009

[19] Coutinho, A.E. and Chapman, K.E. (2011) The Anti-Inflammatory and Immunosuppressive Effects of Glucocorticoids, Recent Developments and Mechanistic Insights. Molecular and Cellular Endocrinology, 335, 2-13.

https://doi.org/10.1016/j.mce.2010.04.005

[20] Bachman, E., Travison, T.G., Basaria, S., Davda, M.N., Guo, W., Li, M., Connor Westfall, J., Bae, H., Gordeuk, V. and Bhasin, S. (2013) Testosterone Induces Erythrocytosis via Increased Erythropoietin and Suppressed Hepcidin: Evidence for a New Erythropoietin/Hemoglobin Set Point. The Journals of Gerontology Series A Biological Sciences and Medical Sciences, 69, 725-735. https://doi.org/10.1093/gerona/glt154

[21] Miočević, O., Cole, C.R., Laughlin, M.J., Buck, R.L., Slowey, P.D. and Shirtcliff, E.A. (2017) Quantitative Lateral Flow Assays for Salivary Biomarker Assessment: A Review. Frontiers in Public Health, 5, 133. https://doi.org/10.3389/fpubh.2017.00133

[22] McHenry, J., Carrier, N., Hull, E. and Kabbaj, M. (2014) Sex Differences in Anxiety and Depression: Role of Testosterone. Frontiers in Neuroendocrinology, 35, 42-57. https://doi.org/10.1016/j.yfrne.2013.09.001

[23] Harman, S.M., Metter, E.J., Tobin, J.D., Pearson, J. and Blackman, M.R. (2001) Longitudinal Effects of Aging on Serum Total and Free Testosterone Levels in Healthy Men. Baltimore Longitudinal Study of Aging. The Journal of Clinical Endocrinology \& Metabolism, 86, 724-731. https://doi.org/10.1210/jcem.86.2.7219

[24] Clifton, S., Macdowall, W., Copas, A.J., Tanton, C., Keevil, B.G., Lee, D.M., Mitchell, K.R., Field, N., Sonnenberg, P., Bancroft, J., Mercer, C.H., Wallace, A.M., Johnson, A.M., Wellings, K. and Wu, F.C. (2016) Salivary Testosterone Levels and Health Status in Men and Women in the British General Population: Findings from the Third National Survey of Sexual Attitudes and Lifestyles (Natsal-3). The Journal of Clinical Endocrinology \& Metabolism, 101, 3939-3951. https://doi.org/10.1210/jc.2016-1669

[25] Nunes, L.A., Mussavira, S. and Bindhu, O.S. (2015) Clinical and Diagnostic Utility of Saliva as a Non-Invasive Diagnostic Fluid: A Systematic Review. Biochemia Medica, 25, 177-192. https://doi.org/10.11613/BM.2015.018 
[26] De Benedittis, G., Massei, R., Nobili, R. and Pieri, A. (1988) The Italian Pain Questionnaire. Pain, 33, 53-62. https://doi.org/10.1016/0304-3959(88)90203-5

[27] Apolone, G. and Mosconi, P. (1998) The Italian SF-36 Health Survey: Translation, Validation and Norming. Journal of Clinical Epidemiology, 51, 1025-1036. https://doi.org/10.1016/S0895-4356(98)00094-8

[28] Aloisi, A.M., Buonocore, M., Merlo, L., Galandra, C., Sotgiu, A., Bacchella, L., Ungaretti, M., Demartini, L. and Bonezzi, C. (2011) Chronic Pain Therapy and Hypothalamic-Pituitary-Adrenal Axis Impairment. Psychoneuroendocrinology, 36, 10321039. https://doi.org/10.1016/j.psyneuen.2010.12.017

[29] Paananen, M., O’Sullivan, P., Straker, L., Beales, D., Coenen, P., Karppinen, J., Pennell, C. and Smith, A. (2015) A Low Cortisol Response to Stress Is Associated with Musculoskeletal Pain Combined with Increased Pain Sensitivity in Young Adults: A Longitudinal Cohort Study. Arthritis Research \& Therapy, 17, Article No. 355. https://doi.org/10.1186/s13075-015-0875-Z

[30] Van Cauter, E., Leproult, R. and Kupfer, D.J. (1996) Effects of Gender and Age on the Levels and Circadian Rhythmicity of Plasma Cortisol. The Journal of Clinical Endocrinology \& Metabolism, 81, 2468-2473. https://doi.org/10.1210/jc.81.7.2468

[31] Horstman, A.M., Dillon, E.L., Urban, R.J. and Sheffield-Moore, M. (2012) The Role of Androgens and Estrogens on Healthy Aging and Longevity. The Journals of Gerontology Series A Biological Sciences and Medical Sciences, 67, 1140-1152. https://doi.org/10.1093/gerona/gls068

[32] Qiu, W.Q., Claunch, J., Kong, J., Nixon, E.E., Fang, J., Li, M., Vangel, M. and Hui, K.K. (2010) The Effects of Acupuncture on the Brain Networks for Emotion and Cognition: An Observation of Gender Differences. Brain Research, 1362, 56-67. https://doi.org/10.1016/j.brainres.2010.09.040 